\title{
Uncloaking globular clusters of the inner Galaxy
}

\author{
Javier Alonso-García ${ }^{1}$, Mario Mateo ${ }^{1}$ and Bodhisattva Sen ${ }^{2}$ \\ ${ }^{1}$ Department of Astronomy, University of Michigan, 500 Church St.-830 Dennison Bldg., \\ Ann Arbor MI 48109, USA \\ email: jalonso,mmateo@umich.com \\ ${ }^{2}$ Department of Statistics, University of Michigan, 1085 South University St.-439 West Hall, \\ Ann Arbor MI 48109, USA \\ email: bodhi@umich.edu
}

\begin{abstract}
We intend to map the differential extinction and remove their effects in a sample of clusters in the direction of the inner Galaxy using a new dereddening technique. These observations and their analyses will let us produce high quality color-magnitude diagrams of these poorly studied clusters that will allow us to determine these clusters' relative ages, distances and chemistry and to address important questions about the formation and the evolution of the inner Galaxy.
\end{abstract}

Keywords. Galaxy: bulge, (Galaxy:) globular clusters: general

\section{Scientific interest and goals}

The age, chemical and kinematic distributions of stellar populations provide powerful constraints on models of the formation and evolution of the Milky Way. The globular clusters constitute a particularly useful case because the stars within individual clusters are coeval and spatially distinct. But a serious limitation in the study of many globular clusters - especially those near the Galactic Center - has been the existence of large absolute and differential extinction by foreground dust. We observed a sample of GCs in the direction of the inner Galaxy and now we can apply a new technique to differentially deredden the cluster photometric data (details in next section). We intend to use such dereddened cluster photometric data to build clean CMDs that, by analysis and comparison with modern stellar evolutionary models, will let us determine the relative mixtures, age sequence, and chemical properties of the cluster populations in the inner Milky Way.

An important by-product of our dereddening process is a map of the differential extinction across a cluster. This will allow us to sample the ISM along numerous low latitude lines of sight, paying special attention to the low mass, low column density clouds of the cold ISM which are normally invisible at IR wavelengths.

\section{The dereddening technique}

After getting the CMD of the globular cluster,

- A ridgeline is obtained applying a non-parametric fitting method (univariate kernel regression).

- The stars in the cluster are moved along the reddening vector until they intersect the ridgeline.

- The shifts of the stars are smoothed using again a non-parametric fitting method (bivariate kernel regression), which provides the value of the magnitude of the extinction in a place of the field. 


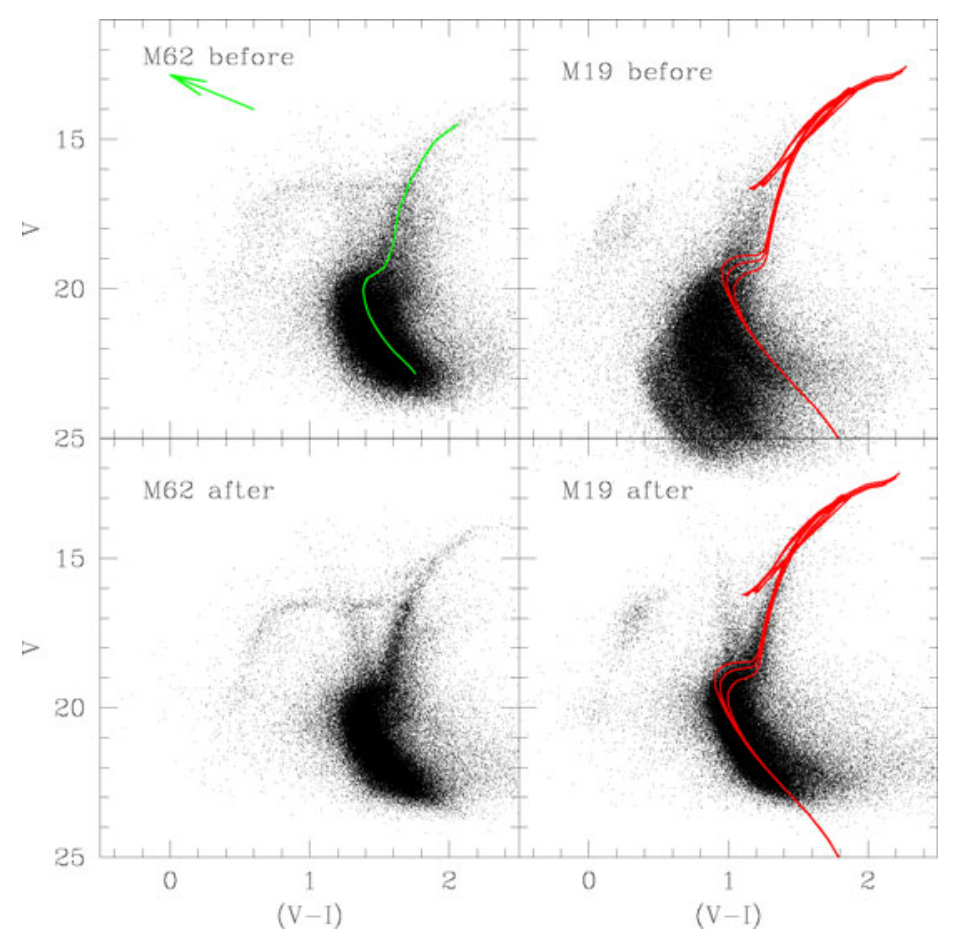

Figure 1. CMDs of M62 (left) and M19 (right) before (up) and after (down) going through our dereddening proccess. We can see that after the effects of the internal reddening have been removed we are able to define much better the different regions of the CMDs and to fit better the stellar models to calculate the characteristics of these clusters. For M62, we have plotted the reddening vector along which the stars are moved until they intersect the ridgeline, also plotted. The width of the MS narrows by a factor of 2 after being dereddened. For M19, we have fitted a series of Padova isochrones with ages 8,10 and 14 Gyrs. After being differentially dereddened we can clearly constrain the age of the cluster to be between 11-14 Gyr and the metallicity in the range $\mathrm{z} \sim 0.001$, while from the original $\mathrm{CMD}$, the age was uncertain by a factor of 2 , and the RGB too indistinct to constrain the metallicity.

This is an iterative process, since after we have corrected for the extinction we can better obtain the cluster ridgeline, which lead us to a better estimation of the extinction value.

\section{Observations and first results}

A sample of 25 globular clusters located toward the center of the Milky Way were observed in B, V and I using the IMACS imager (FOV $15.4^{\prime} \times 15.4^{\prime}$ ) on the LCO Baade $6.5 \mathrm{~m}$ telescope. We have already extracted the photometry and astrometry of the observed globular clusters, and we have started to apply our extinction correction method in some of them. In Figure 1 we can see examples of the clusters' CMDs before and after going through our dereddening proccess. The undereddened CMD more clearly shows key features of the cluster CMD (BHB, SGB, narrow RGB) that were indistinct in the original CMD. This improvement allows us to much more precisely estimate the cluster characteristics. 